\title{
Neuropatía auditiva, diagnóstico y manejo audiológico
}

\section{Auditory neuropathy / dyssychrony, assessment and audiologic management}

\author{
Oscar Cañete $\mathrm{S}^{1}$.
}

\begin{abstract}
RESUMEN
Los hallazgos clínicos que definen una neuropatía/des sincronía (NA/DA) son la integridad de las células ciliadas evidenciado, mediante la presencia de emisiones otoacústicas evocadas (EOAe) y/o la presencia de potenciales microfónicos cocleares (PMIC), junto con la ausencia de actividad neural evocada a nivel del VIII par craneano (potencial de acción compuesto) y tronco cerebral (potencial evocado auditivo de tronco (PEAT)). Las condiciones clínicas relacionadas con una NA/DA incluyen a la hiperbilirrubinemia, enfermedades neurovegetativas (ejemplo ataxia de Friedreich), síndrome de Charcot-Marie-Tooth así como otras neuropatías sensoriomotoras, desórdenes mitocondriales y neuropatías isquémica-hipóxicas como resultados de asfixia.

El desarrollo de las habilidades auditivas y comunicacionales pueden estar comprometidas en forma importante en los niños con aparición prelingual de una NAVDA, mayor es aún este compromiso al no existir un perfil pronóstico preestablecido del desarrollo y resultados en el tratamiento de este desorden. En la actualidad existen múltiples estudios en donde se ha observado que hasta 50\% de los pacientes con NAVDA presentan algún grado de beneficio al usar audífonos por lo que se sugiere que sistemas de amplificación como los audífonos o incluso implante coclear deberían ser el primer paso en el proceso de (re)habilitación.

Palabras clave: Neuropatía auditiva, des sincronía auditiva, desorden del espectro de neuropatía auditiva, hipoacusia neural, hipoacusia en neonatos de riesgo, potenciales evocados de tronco.
\end{abstract}

\begin{abstract}
The clinical findings that define an auditory neuropathy/dyssynchrony (AN/Dys) are the integrity of the outer hair cells demonstrated by the presence of evoked otoacoustics emissions (OAEe) and/or the presence of cochlear microphonic potential (CMP) along with the absence of neural activity evoked at level of VIII nerve (action potential compound) and brainstem (Auditory Brainstem Response, ABR). The clinical conditions related to an AN they include hiperbilirrubinemia, neurodegenerative diseases (eg ataxia of Friedreich), CharcotMarie-Tooth syndrome as well as other sensoriomotor neuropathies, mitocondrial disorders and hypoxic neuropathy as perinatal asphyxia.

The development of the auditory and communicational abilities can be compromise in children with prelingual onset of an AN/Dys, the development and results of treatment of this disorder is still this commitment when not existing a profile prognosis. At the present time multiple studies have been observed that until a $50 \%$ of the patients with AN present some degree of benefit using hearing aids, reason why it suggests amplification systems as the hearing aids or even cochlear implant would have to be the first step in the process of (re) habilitation of these patients.
\end{abstract}

Key words: Auditory neuropathy, Neural hearing loss, Auditory evoked potentials, Auditory Dyssynchrony. Hearing loss in high risk newborns.

1 Tecnólogo Médico, Servicio de Otorrinolaringología, Hospital Padre Hurtado. 


\section{INTRODUCCIÓN}

El término "neuropatía auditiva" (NA) fue utilizado por primera vez por audiólogos y médicos como Starr, Picton, Sininger, Hood y Berlin en la década de los 90. Estos describieron hallazgos en pacientes que presentaban una pérdida auditiva con la presencia de emisiones otoacústicas evocadas (EOAe) y ausencia 0 anormalidad severa de los potenciales evocados auditivos de tronco (PEAT). La mayoría de estos pacientes presentaban dificultades en la comprensión del habla particularmente en presencia de ruido'.

En la actualidad es posible la diferenciación de un NA de una pérdida auditiva coclear gracias al uso sistemático de los PEAT y de las EOAe como herramientas de evaluación audiológica. Se ha sugerido el término des sincronía auditiva, pérdida auditiva neural, entre otras denominaciones ${ }^{2}$. Recientemente en la conferencia internacional celebrada en Como, Italia (Newborn Hearing Screening Conference, junio 2008) se estableció el término "Desorden del espectro de neuropatía auditiva (DENA)" tratando de describir su heterogeneidad y naturaleza multifactorial ${ }^{3}$. En la presente revisión se utilizará el término neuropatía auditiva/des sincronía (NA/DA) considerando que son términos ampliamente conocidos y el cambio de nomenclatura aún no se ha masificado.

El término NA/DA permite describir un desorden auditivo en el cual la función de las células ciliadas externas (CCE) se encuentra conservada no así la transmisión aferente de la vía auditiva ${ }^{4}$. Esta indemnidad puede ser demostrada mediante EOAe o con la presencia del PMIC, mientras que las alteraciones en la transmisión neural o sincronía se manifiestan en la ausencia de actividad neural (potencial del acción del nervio, PA) o PEAT. La audición periférica en estos pacientes (umbrales auditivos) puede situarse entre rangos de normalidad hasta pérdidas auditivas profundas" ${ }^{1}$.

\section{Patrones de NA/DA}

Los hallazgos clínicos que definen una NA/DA son la integridad de las células ciliadas mediante la presencia de EOAe y/o la presencia de PMIC junto con la inhabilidad de registrar la actividad neural evocada a nivel del VIII par craneano y tronco cerebral. Desde el punto de vista electrofisiológico este perfil es denominado "retrococlear", sin embargo el sitio de origen exacto y mecanismo patológicos involucrados aún no han sido determinados de todo. Otro hallazgo clínico consistente de una NA/DA es la presencia de una hipoacusia permanente 0 fluctuante, estudio imagenológico normal, ausencia de reflejo acústico y déficits en la discriminación y/o percepción auditiva la cual no está en relación con los umbrales audiométricos ${ }^{5,6}$.

La aparente buena funcionalidad de las células ciliadas distingue a las NA de una pérdida auditiva sensorial o sensorioneural. En la mayoría de los casos de pérdidas adquiridas o congénitas, las CCE se encuentran más afectadas que las células ciliadas internas (CCl) así como pueden estar ambas alteradas o presentar un daño en su desarrollo (como las displasias cocleares). Esta aparente buena funcionalidad de las CCE en conjunción con una pérdida auditiva importante así como habilidades de discriminación descendidas, hacen que las características de la NA/DA sean paradójicas ${ }^{4}$.

La disminución de la sensibilidad auditiva puede ser el resultado de alteraciones en diferentes sitios a través de la vía auditiva periférica o central. La forma más común de una pérdida auditiva permanente es el resultado de una anormalidad a nivel de la cóclea, pudiéndose relacionar con una pérdida 0 mal función de la $\mathrm{CCl}$, pérdida $0 \mathrm{mal}$ función de la amplificación coclear (el cual se piensa que reside en la electromotilidad de las CCE que depende de prestina y proporciona un aumento en la sensibilidad auditiva de hasta $60 \mathrm{~dB})^{7} 0$ la disrupción de la $\mathrm{CCl}$ conocido como potencial endococlear. El déficit auditivo coclear, en general se refiere a una pérdida sensorial, afección del oído interno, que afecta a las $\mathrm{CC}^{4,5}$.

\section{Etiología y patología}

Se han propuesto posibles explicaciones de los resultados electrofisiológicos y conductuales: una disfunción que afecta la sinapsis entre las $\mathrm{CCl}$ y las fibras nerviosas auditivas tipo 1, una falla en las transmisiones de las $\mathrm{CCl}$, desorden en las células del ganglio espiral o desmielinización de la vía auditiva 0 daño específico en las poblaciones neuronales de la vía auditiva o desmielinización del nervio auditivo, se 
han propuesto como posible explicación de los resultados audiométricos y elecrofisiológicos ${ }^{4,5}$.

Las condiciones clínicas relacionadas con una NA/DA incluyen a la hiperbilirrubinemia, enfermedades neurovegetativas (ejemplo ataxia de Friedreich), síndrome de Charcot-Marie-Tooth así como otras neuropatías sensoriomotoras, desórdenes mitocondriales, neuropatias isquémicahipóxicas resultados de asfixia ${ }^{4-6,8}$.

\section{Hiperbilirrubinemia}

Los niveles excesivos de bilirrubina (producto del metabolismo de los glóbulos rojos), la cual es asociada a la inmadurez hepática en los recién nacidos (RN) puede llegar a ser toxica para el sistema nervioso central (SNC), produciendo daños neurológicos importantes ${ }^{2}$. La mayoría de los RN en sus primeros días de vida, transitoriamente alcanzan niveles elevados de bilirrubina no conjugada en sangre, concentraciones cercanas 0 superiores a $20 \mathrm{mg} / \mathrm{dl}$ de bilirrubina no conjugada podría tener efectos tóxicos produciendo una pérdida auditiva ${ }^{9}$, cuando estos valores son superados se han descrito anormalidades tanto temporales como permanentes en IOS PEATs, incluyendo la elevación del umbral electrofisiológico del PEAT y latencias prolongadas interondas I-V ${ }^{10}$, esto sugiere que tanto el sistema auditivo periférico como central son altamente vulnerables a esta noxa ${ }^{5}$. Los núcleos del tronco incluyendo los núcleos cocleares, complejo olivar superior, lemnisco lateral, cuerpo trapezoide y colículo inferior pueden verse afectados con esta condición ${ }^{11}$.

Algunos de los primeros casos de NA se presentaron en niños y adolescentes que presentaron una hiperbilurribinemia y Kernicterus neonatal. La hiperbilirrubinemia da cuenta de la mitad de las causas de NA/DA en algunas series ${ }^{4}$. No debemos olvidar que para este cuadro existe una remisión espontánea de los síntomas $^{4,12}$. No existen factores pronósticos para tal remisión, ocurriendo ésta en un pequeño número de casos reportados. Cabe señalar que la hiperbilirrubinemia no sólo es un factor de riesgo para una NA/DA sino también de hipoacusia sensorial ${ }^{4,8}$.

\section{Agentes infecciosos}

Los casos de NA/DA relacionados con causas infecciosas ha sido sugerido en un número peque- ño pero no despreciable de casos en la literatura reciente. Se ha podido observar en algunas series que procesos infecciosos virales estaban involucrados en el $10 \%$ de 67 pacientes que presentaron NA/DA5. Detalles específicos sobre la etiología no fueron presentados en estos sujetos, pero existen otros estudios reportados como sarampión y meningitis, las cuales pueden ser asociadas a una NA ${ }^{5}$.

\section{Neuropatías sensoriomotoras hereditarias y otros desórdenes neurodegenerativos}

Una NA/DA puede presentarse como parte de un desorden neuropático generalizado. Las neuropatías motoras y sensoriales como el síndrome Charcot-Marie-Tooth (Tipo I \& II) muestran una alta proporción de casos de NA/DA. El síndrome de Charcot-Marie-Tooth es un desorden genético el cual involucra una degeneración de la vaina de mielina, la cual se relaciona con una anormalidad en la proteína 22 (PMP22) de la mielina cuyo gen se ubica en el cromosoma $17 \mathrm{p} 11.2$ o en la maduración del gen MPZ (1q 22.3). La pérdida de los axones de las porciones distales de los nervios periféricos también ha sido reportado en esta condición. Las respuestas de los PEAT pueden estar ausentes 0 ser muy anormales ${ }^{1,5}$.

En una serie de 11 pacientes (niños y adultos) que presentan NA/DA, en que la mayoría de ellos presentaban signos clínicos y electrofisiológicos de otras neuropatías en otros nervios periféricos ${ }^{5}$. Se ha podido observar síntomas de NA/DA en pacientes que presentan el síndrome de Charcot-Marie-Tooh tipo I \& II ${ }^{1,2,4}$ así como también en otras neuropatías motoras hereditarias circunscritas a grupos familiares específicos.

\section{Neuropatía isquémica-hipóxica}

Recién nacidos de prematurez extrema presentan un alto riesgo para un número importante de secuelas neurológicas, incluyendo neuropatías visuales y auditivas. Los eventos isquémicoshipóxicos pueden afectar las áreas corticales y subcorticales de las vías auditivas así como en la cóclea ${ }^{4}$. 


\section{Otros de origen genético}

La mutación DFNB9 produce una pérdida auditiva prelingual (autosómica recesiva) muestra patrones típicos de una NA, es decir, ausencia de ondas en el PEAT en presencia de EOAs, las cuales pueden deteriorarse en el tiempo. Esto sería causa de una mutación del gen OTOF, el cual codifica a la proteína otoferlina. Esta proteína se encuentra presente en la cóclea, vestíbulo y cerebro ${ }^{12,13}$. Dentro de la cóclea este gen se expresa principalmente en la $\mathrm{CCl}$ y en menor proporción en las CCE. Esta corresponde a una proteína citosólica que estaría involucrada en la fusión de las vesículas sinápticas en la membrana plasmática mediada por $\mathrm{Ca}++$. Esta deficiencia en los canales de $\mathrm{Ca}++$ (al menos en modelos animales) se caracteriza por el bloqueo completo de la transmisión sináptica de las $\mathrm{CCI}$, asociándose a una reducción de las neuronas del ganglio espiral. En contraste las CCE a nivel basal de la cóclea preservarían su morfología. Debido a lo anterior se postula que la lesión primaria en una NA/DA secundaria a una mutación de OTOF sería a nivel de las CCI siendo también las CCE afectadas pero en menor medida debido a la menor expresión de este gen, siendo ésta la explicación de la presencia de EOA provocadas en estos pacientes ${ }^{12-14}$. Una deficiencia en las zonas activas presinápticas de las CCl afectaría la rapidez de la exocitosis de vesículas sinápticas y sincronía de la activación sináptica de las neuronas del ganglio espiral. Datos obtenidos en modelos animales (ratones) sugieren que la disfunción sináptica causada principalmente por una disminución en las sinapsis de las CCI, podría comprometer la sincronización de las señales y habilidad de las neuronas del tronco para detectar diferencias interaurales del tiempo, función que recae principalmente en el tiempo de liberación de las vesículas sinápticas en la sinapsis de las $\mathrm{CCI}^{12}$.

\section{Neuropatía auditiva sensible a temperatura}

Existen 3 casos reportados en niños que presentaron pérdidas auditivas severas a profundas junto con una pérdida de los PEATs, con mantención de EOAs en estado febril ${ }^{6}$. Este efecto dependiente de temperatura es similar a los efectos de exacerbación visto en pacientes que presentan esclerosis múltiple cuando la temperatura corporal aumenta, esto sugiriera que los síntomas auditivos son producto de un desorden de desmielinización (con el concomitante bloqueo de la conducción nerviosa) ${ }^{4,15}$. Así mismo se han descrito pacientes con mutaciones en el gen OTOF que presentan características descritas con anterioridad por Starr ${ }^{16}$.

\section{Epidemiología de la neuropatía auditiva}

\section{Edad de aparición de los síntomas}

Edad de aparición de una pérdida auditiva producto de una NA/DA se puede clasificar en 2 grupos distintivos; aquellos que presentan los síntomas desde la infancia, y aquellos quienes desarrollan tal condición en su adolescencia 0 adultez tempra$\mathrm{na}^{5,17}$. En la actualidad gracias al uso de técnicas como el PEAT y EOAs es posible diagnosticar apropiadamente las NA/DA en la población pediátrica (generalmente gracias a la implementación de programas de pesquisa auditiva $)^{5,12}$.

\section{Prevalencia}

Debido a que las causas de una NA/DA son variadas, es difícil estimar la prevalencia de este desorden auditivo además existen pocos estudios a gran escala que metodológicamente estén bien realizados (muestreo, protocolos de evaluación, etc.) que permitan realizar estimaciones más precisas al respecto.

En la actualidad las estimaciones se basan principalmente en la población que presenta uno 0 más factores de riesgo de presentar una pérdida auditiva ${ }^{4}$. Se ha estimado en algunas investigaciones que $1 \mathrm{de}$ cada 200 pacientes con alguna pérdida auditiva presentan hallazgos en el PEAT inconsistentes con los tonos puros del audiograma 4 . Algunos autores han determinado que la prevalencia de una NA/DA es de 1 en 433 en un grupo de 5.199 infantes que presentaron factores de riesgo auditivo al momento de nacer, éstos presentaron ausencia de PEAT, con presencia de EOA y $\mathrm{PMIC}^{4}$. En una cohorte de 109 niños con pérdida auditiva permanente de al menos un grado moderado (identificados en el programa de pesquisa auditiva de recién nacidos de riesgo) se encontró la presencia de una NA en $11 \%$ del total. Algunos investigadores han observado series en donde 5 de 111 niños con una pérdida auditiva permanente pasaron las EOA, siendo diagnosticada una NA/DA en 2 casos de éstos. La presencia de una pérdida auditiva bilateral de al menos grado moderado se encuentra presente en 1,4 por cada 1.000 nacidos vivos ${ }^{4}$. Por lo tanto la incidencia de una NA/DA, utilizando estimaciones conservadoras, sería 
de 1,4 por 10.000 nacidos vivos. Utilizando las estimaciones de Rance y cols (1999), una NA/DA podría presentarse en 2,3 por 1.000 recién nacidos con factores de riesgo de hipoacusia. Estos datos sugieren que si se utilizan sólo las EOA en la etapa de pesquisa en recién nacidos que presenten factores de riesgo, cerca de $11 \%$ de las hipoacusias permanentes no serían diagnosticadas ${ }^{4}$ (ver Tablas 1 y 2 ).

Es importante recordar que el diagnóstico de este desorden es relativamente reciente por lo que el subdiagnóstico es una realidad. Estimaciones generales establecen una prevalencia entre $0,23 \%$ y $0,94 \%$ en niños con factores de riesgo de pérdida auditiva. Entre los niños que presentan una pérdida auditiva permanente la prevalencia puede alcanzar entre $7 \%$ a $11 \%{ }^{9}$ y en el caso de los recién nacidos que egresan de la unidad de cuidados intensivos entre $4 \%$ y $6 \%{ }^{18}$.

\section{HALLAZGOS AUDIOLÓGICOS Y PSICOACÚSTICOS}

Una NA/DA se caracteriza por los siguientes hallazgos audiológicos:

1. EOAe presentes, basta con la presencia de éstas en una sola oportunidad. Se ha visto que pueden desaparecer en el transcurso del tiempo o con el uso de audífonos ${ }^{4,5,19,20}$. Es importante considerar que se ha visto la ausencia de EOAs hasta en $30 \%$ de los oídos confirmados con una NA/DA. En relación con este ultimo punto no se ha encontrado una significancia estadística entre la presencia 0 ausencia de EOAs y los umbrales conductuales en niños con NA/DA ${ }^{20}$.

2. PEAT ausente 0 marcadamente anormal junto con la presencia de PMIC (la definición de anormal se encuentra aún por determinar, pero se puede considerar a latencias absolutas 0 interonda $>2$ desviaciones estándar para la edad del paciente, amplitudes significativamente menores a lo esperado, con morfología de las ondas marcadamente anormales. Esta definición debe ser utilizada con cautela debido a que hipoacusias severas pueden presentar estas características ${ }^{4,5,19,20}$.

3. Reflejos acústicos (estapediano) ausentes, ipsilaterales y contralaterales ${ }^{1,4-6,19,21}$.

Debido a que en muchos pacientes los resultados pueden variar en el tiempo, es recomendable hacer un seguimiento de éstos, evaluando cada caso en particu-

Tabla 1. Prevalencia de una NA/DA en niños con una pérdida auditiva permanente

\begin{tabular}{|lccc|}
\hline Estudio & $\begin{array}{c}\text { Pacientes con pérdida } \\
\text { auditiva }\end{array}$ & $\begin{array}{c}\text { Pacientes con } \\
\text { neuropatía auditiva }\end{array}$ & $\%$ del total \\
\hline Kraus et al 1984 & 48 & 7 & 14,6 \\
Cone-Wesson et al 2000 & 56 & 3 & 5,3 \\
Rance et al 1999 & 109 & 12 & 11 \\
Total & 213 & 22 & 10,3 \\
\hline
\end{tabular}

Fuente: Rance G. Auditory Neuropathy/Dyssynchrony and it's Perceptual Consequences. Trends Amplif 2005; 9(1): 1-43.

Tabla 2. Estimación de la prevalencia de NA/DA en infantes con factores de riesgo

\begin{tabular}{|lccc|}
\hline Estudio & $\begin{array}{c}\text { Recién nacidos } \\
\text { con riesgo }\end{array}$ & $\begin{array}{c}\text { Positivo para } \\
\text { neuropatía auditiva }\end{array}$ & $\%$ del total \\
\hline Rance et al, 1999 & 5.199 & 12 & 0,23 \\
Stein et al, 1996 & 100 & 4 & 4,0 \\
Psarommatis et al, 1997 & 102 & 12 & 1,96 \\
Total & 5.401 & 28 & 0,51 \\
\hline
\end{tabular}

Fuente: Rance G. Auditory Neuropathy/Dyssynchrony and it's Perceptual Consequences. Trends Amplif 2005; 9(1): 1-43. 
lar en forma previa. Se ha podido establecer otras pruebas complementarias que nos ayudarían a establecer la presencia de una NA/DA, éstas son: 1) Supresión eferente de las EOA ausente (indica la integridad del sistema auditivo eferente). 2) Efecto en prueba de MLD (masking level diference) ausente. 3) Discriminación auditiva, no correlacionada con los umbrales audiométricos, también se ha visto un desempeño descendido en presencia de ruido de fondo $0^{4,5,20,21}$.

Es importante tener cuidado al momento de interpretar los resultados de un PEAT en recién nacidos de pretérmino menores de 34 semanas de gestación, esto debido al efecto de maduración que puede afectar los resultados del PEAT. Mientras sea posible es adecuado repetir este procedimiento antes de realizar un diagnóstico definitivo particularmente en niños con riesgo auditivo. Al interpretar los resultados de un PEAT con trazado anormal pero replicable es adecuado establecer el criterio de "anormal" debido que éste puede sufrir variaciones entre los diferentes centros. Es así como se sugiere que los parámetros que describen la morfología de una onda sean de carácter cuantitativo, por ejemplo una baja amplitud de onda $\mathrm{V}$, con aumento de latencias y sin la presencia de otras ondas. Idealmente el PEAT debería ser repetido a los 3 meses de edad y luego a los 6-8 meses de edad para evaluar el posible efecto neuromaduracional $^{2}$ (ver Figura 1).
En la actualidad ha surgido la electrococleografía (ECohG) como una herramienta de apoyo diagnóstico en pacientes con NA/DA. Esta es una medición de los potenciales eléctricos que son generados en el oído interno como resultado a la estimulación sonora. Se pueden distinguir 3 respuestas; PMIC, potencial de sumación (PS) y potencial de acción compuesto del nervio auditivo (PAC). El PMIC tiene la capacidad de reflejar la integridad de las CCE, lo que jugaría un rol preponderante en la identificación de oídos con una NA. Se ha reportado que casi la mitad de los individuos con NA/DA fueron adecuadamente diagnosticados con la presencia del PMC a pesar de la ausencia de EOAs ${ }^{20}$. La utilización del PMIC y las EOAs en conjunto son elementos objetivos que permiten indicar la funcionalidad de las CC en pacientes con ausencia de PEAT, permitiendo así una mayor precisión en el diagnóstico ${ }^{12}$. La utilización de la ECohG ha permitido postular una clasificación de la NA/DA, es así como se ha señalado la existencia de un tipo pre y postsináptico, esto tendría implicancia al momento de considerar un implante coclear como posible tratamiento ${ }^{18}$.

A pesar de que exista una ausencia total del PEAT, se estima que cerca del $7 \%$ de los infantes 0 niños actuaran como si no presentaran ningún problema auditivo, incluso podrían no presentar ningún problema del habla o lenguaje ${ }^{19}$.

Se sugiere la utilización de polaridad negativa y positiva del estímulo en la evaluación con PEAT,

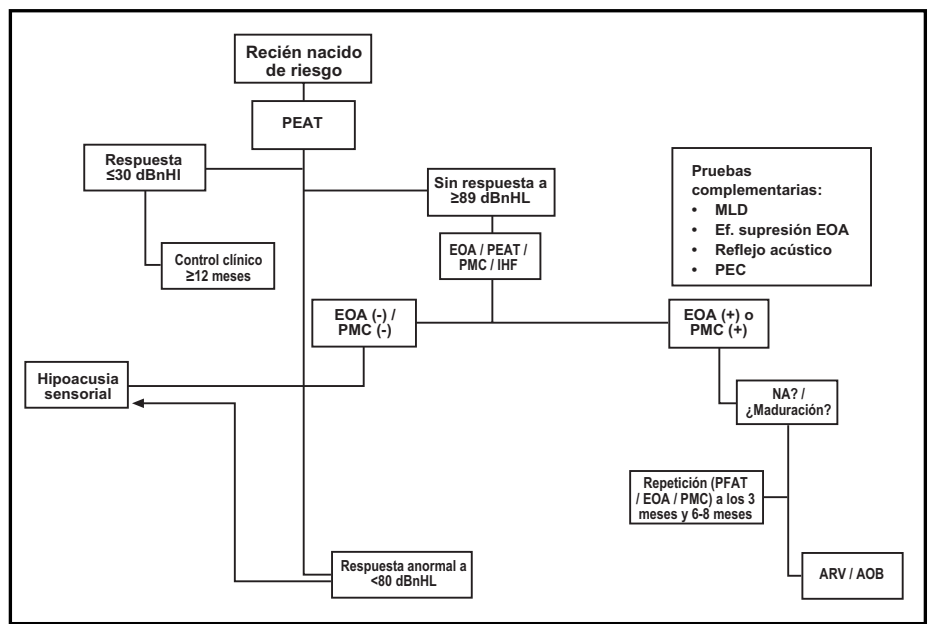

Figura 1. Flujograma propuesto para el diagnóstico de una neuropatía auditiva.

PEAT: Potencial evocado auditivo de tronco. EOA: Emisiones otoacústicas. PMC: Potencial microfónico coclear. IHF: Impedanciometría de alta frecuencia. MLD: Masking level difference. PEC: Potenciales evocados de corteza. ARV/AOB: Audiometría de refuerzo visual / Audiometría observacional. 
especialmente si una NA/DA es sospechada, esto ayudaría a revelar la presencia del PMIC ${ }^{4,19}$. Esto gracias a que la polaridad del estímulo determina la polaridad del registro (PMIC), permitiendo de este modo observar este potencial ${ }^{19}$ (ver Figura 2).

En relación al audiograma no existe un perfil determinado que caracterice a un paciente con NA/ $D A$, ya que éste puede ser desde una pérdida moderada a profunda. Los primeros reportes de una NA/DA describieron la pérdida auditiva de tipo ascendente para las frecuencias bajas ${ }^{1,5}$. La discriminación auditiva sin correlación con los umbrales auditivos es un hecho característico de una NA/DA así como es hallazgo importante en el establecimiento del tratamiento adecuado $0^{4,5}$.

Utilizando pruebas psicoacústicas más específicas se pudo comprender de mejor forma la deficiencia en la discriminación que se observada en este desorden. Es así como las habilidades temporales en estos individuos se encuentran marcadamente disminuidas, lo que ayuda a que la capacidad de discriminación auditiva sea deficiente así como sin correlación con el grado de hipoacusia obtenida en el audiograma. Este déficit en el procesamiento temporal sería resultado de la de sincronía de la actividad neural a nivel del nervio auditivo ${ }^{23,24}$.

\section{Potenciales evocados corticales (PEC)}

Debido a que una de las principales características de una NA/DA es la ausencia 0 anormalidad de los PEAT, podríamos esperar que los potenciales evocados de origen más central también deberían estar afectados

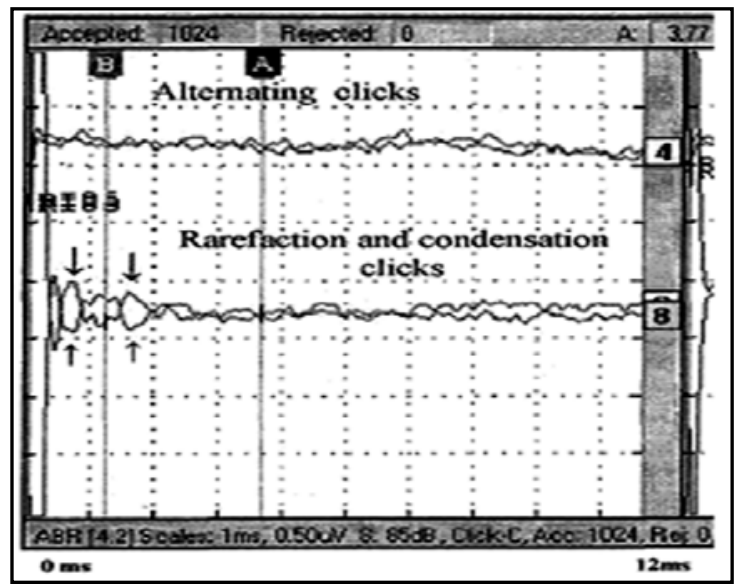

tal es el caso de los potenciales de latencia media (PLM) y PEC. Sin embargo se han encontrado resultados de los complejos N1/P1, P2/N2 así como el potencial de disparidad o mismatch negativity dentro de los rangos normales (latencia, amplitud) ${ }^{25}$. Se han encontrado PEC en niños con NA/DA de características similares a aquellos encontrados en grupos (cohorte de edad) tanto en niños con audición normal como con hipoacusia sensorioneural $1^{1,4,5}$.

Es bien conocida la poca correlación entre la habilidad de discriminación y los umbrales de tonos puros en pacientes que presentan una NA/DA. Sin embargo se ha podido establecer que los potenciales de corteza se pueden obtener en al menos $50 \%$ de los niños con NA/DA ${ }^{12}$. Los potenciales de corteza como N1/P1, P2/N2 pueden ser una herramienta útil para predecir el rendimiento en las pruebas de discriminación del habla en individuos con NA/DA. La presencia de estos potenciales así como de una amplitud adecuada ha demostrado poseer una buena correlación con las habilidades de discriminación a formato abierto. La ausencia de estos potenciales indica una habilidad de percepción del habla bastante pobre. Si se logra replicar estos resultados en grupos mayores de pacientes esto podría proporcionar importante información relacionada con severidad y opciones en el manejo y tratamiento de este desorden ${ }^{26}$.

\section{MANEJO}

El desarrollo de las habilidades auditivas y comunicacionales pueden estar comprometidas en forma impor-

Figura 2. Ondas de un PEAT característico de una NA/DA. Trazado superior: Sin respuesta (estímulo click) Trazado inferior: PMIC evidenciado utilizando polaridad de rarefacción y condensación (separadamente). EI PMIC es el reflejo de la actividad de las CCE y CCI. Este potencial se observa mientras el estímulo está presente. La polaridad del estímulo determina la polaridad del registro (PMIC), por lo tanto al presentar un estímulo utilizando rarefacción (negativo) se observa una onda negativa a diferencia de condensación (positivo) en donde ésta es positiva. Este hecho no se observa en las respuestas neurales (replicación de la polaridad). 
tante en los niños con aparición prelingual de una NA/ DA, mayor es aún este compromiso al no existir un perfil pronóstico preestablecido del desarrollo y resultados en el tratamiento de una NA/DA. Debido a esto cada programa de tratamiento debe ser individual y modificado de acuerdo al progreso de cada paciente ${ }^{12}$.

En la actualidad aún existe cierto grado de controversia en relación con el tratamiento más adecuado para una NA/DA, principalmente con el uso de algún sistema de amplificación como son el caso de audífonos, sistema FM, implantes cocleares ${ }^{13}$, antiguamente existía una gran reticencia al uso de sistemas de amplificación en pacientes que presentaban NA/DA esto principalmente a preocupación generada al utilizar un sistema de amplificación con evidencia de funcionamiento de las CCE pudiendo provocar una potencial daño por ruido ${ }^{12}$. En la actualidad esto ha ido cambiando ya que existen múltiples estudios en donde se ha observado que hasta $50 \%$ de los pacientes con NA/DA presentan algún grado de beneficio al usar audífonos, el cual es similar a aquellos pacientes que presentan una pérdida sensorial por lo que se sugiere que sistemas de amplificación como los audífonos deberían ser el primer paso en el proceso de (re)habilitación ${ }^{12,20}$. Se ha demostrado que no existe una relación significativa entre los niveles de audición de un oído con 0 sin EOAs o entre el desempeño en la discriminación del habla y la presencia 0 no de las EOAs $^{12}$. Sin embargo no es posible establecer relación entre los umbrales auditivos del audiograma y de la discriminación auditiva con el resultado esperado con el uso de audífono.
Se ha señalado la necesidad de audífonos que presenten un tipo de procesamiento del habla especial (por ejemplo, baja ganancia, compresión de rango dinámico), sistema el cual sería necesario en este tipo de pacientes ${ }^{12}$. Junto con ayudar a hacer audible los sonidos, se sugiere que éste ayude a compensar el déficit en el análisis temporal ${ }^{27}$. Se sugiere que los pacientes que presenten umbrales auditivos mayores 0 iguales a 25 decibeles $\mathrm{HL}(\mathrm{dB})$ deberían ser implementados con audífonos siempre en el contexto de un programa integral de rehabilitación aural ${ }^{4}$. El uso de los audífonos ayudaría al paciente al acceso de los elementos del habla y/o mejorando la sincronización neural, estimulando todas las neuronas disponibles ${ }^{12}$. Así mismo los sistemas FM entregarían una opción de bajo riesgo con el potencial beneficio de la mejoría relación señal/ruido.

El implante coclear (IC) se consideraría como el paso final en el tratamiento de estos pacientes, esto dirigido a restaurar el procesamiento comprometido de la información auditiva. En la actualidad existe evidencia sobre la ventaja de la implantación en pacientes con $N A / D A^{28}$. Niños con una actividad neural anormal debido a un daño de las CCI con conservación de las CCE pueden desempeñarse pobremente con los audífonos convencionales, esto incluso puede suceder cuando no existe una NA. Es posible que la percepción del habla se encuentre afectada debido a una anormal sintonización coclear ${ }^{28}$.

Diferentes estudios señalan la utilidad del implante coclear en este tipo de pacientes sin embargo los resultados pueden ser diferentes de un

Tabla 3. Resultados audiológicos esperados en pacientes que presentan una NA/DA

\begin{tabular}{|ll|}
\hline Prueba & Resultado \\
\hline Umbrales de tonos puros & $\begin{array}{l}\text { Desde audición normal a pérdida severa/profunda } \\
\text { (cualquier configuración; pudiendo ser esta asimétrica) } \\
\text { Variable, desde levemente disminuida hasta muy } \\
\text { Discriminación sin ruido }\end{array}$ \\
$\begin{array}{l}\text { comprometida } \\
\text { Reflejo acústico }\end{array}$ & Ausente \\
$-\quad$ Ipsilateral & Ausente \\
$-\quad$ Contralateral & Presente (inversión de la polaridad del estímulo) \\
Potencial microfónico coclear & Ausente o anormal \\
PEAT & Anormal (cercano a 0 dB) \\
Nivel de diferencia del enmascaramiento (MLD) & Sin supresión \\
Supresión eferente de las emisiones otoacústicas (EOAt) & Generalmente deficiente \\
Discriminación en ruido & \\
\hline
\end{tabular}


mejoramiento importante en la discriminación auditiva a formato abierto a resultados discretos 0 simplemente sin resultados ${ }^{29}$.

En relación a este último punto se ha observado a través de la ECoghG y PEAT generados mediante una estimulación eléctrica directa, a nivel de la ventana redonda pueden explicar la variabilidad de los beneficios de un IC y servir de utilidad como indicador pronóstico del éxito de éste. Se ha observado en niños diagnosticados con NA/DA, que sólo en aquellos que presentaron un ECoghG y PEAT robusto adquirieron un buen reconocimiento del habla con el IC, mientras en que los que presentaron un bajo reconocimiento del habla mostraban respuestas anormales (ECoghG y PEAT) ${ }^{30}$.

\section{CONCLUSIONES}

Una NA/DA puede afectar a un número importante de niños con una hipoacusia congénita. La naturaleza heterogénea de este desorden, los diferentes hallazgos clínicos y variabilidad en los resultados con los distintos tratamientos hacen que sea necesario la aplicación de diferentes opciones de manejo. Si bien es cierto una NA/DA se presentaría en un número reducido de pacientes, en la mayoría de estos casos se presentan dificultades comunicativas importantes, lo que afecta la calidad de vida de quien la padece.

Debido a la implementación de programas de pesquisa auditiva en recién nacidos de riesgo en nuestro país no es de extrañarse de la aparición de los primeros casos de individuos que presentan resultados audiológicos contradictorios o pocos concluyentes, esto nos hace considerar la posibilidad de la presencia de una NA/DA, en especial en pacientes que presenten uno 0 más factores de riesgo. Es así como es recomendable el estudio mas profundo de pacientes que presentan estos factores de riesgo al momento de nacer, por ejemplo hiperbilirrubinemia, asfixia, etc. En este tipo de pacientes no bastaría sólo un PEAT ausente 0 anormal para el diagnóstico de hipoacusia, la evaluación con EOAs, y reflejo acústico sería de mucha utilidad para descartar la presencia de una NA $\mathrm{DA}$, esto debido a que el manejo y tratamiento debe ser planificado y monitorizado con precaución.

Una pérdida auditiva debido a una NA/DA presenta un sinnúmero de retos para los profesionales que trabajan en el área de audiología pediátrica los cuales reciben niños tan pequeños como lo son los menores de seis meses. Los umbrales auditivos conductuales no pueden ser predecidos por los métodos de evaluación electrofisiológicos, pudiendo presentarse umbrales normales hasta pérdidas auditivas profundas. Así mismo el impacto de una NA/DA en la habilidad de un niño para utilizar su audición residual tampoco puede ser establecida por sus umbrales auditivos conductuales.

Aún queda mucho por conocer sobre las NA/DA sobre todo en relación con las patologías que podrían producir este desorden así como las alteraciones psicoacústicas producidas, efectos sobre pruebas electrofisiológicas así como la correlación de éstas con una NA/DA.

\section{BIBLIOGRAFÍA}

1. Starr A, Picton T, Sinninger Y, Hood L, Berlin Ch. Auditory Neuropathy. Brain 1996; 119: 741-53.

2. King AM, Purdy SC, Dillon H, Sharma M, Pearce W. Australian Hearing Protocols for the Audiological Management of Infants Who Have Auditory Neuropathy. Australian and New Zealand Journal of Audiology 2005; 27: 69-77.

3. Roush P. Auditory neuropathy spectrum disorder: Evaluation and management. Hear J 2008; 61: 11.

4. Cone-Wesson B, Rance G. Auditory Neuropathy: a brief review. Curr Opin Otolaryngol Head Neck Surg 2000; 8: 421-5.

5. Rance G. Auditory Neuropathy/Dys-synchrony and it's Perceptual Consequences. Trends Amplif 2005; 9: 1-43.

6. Rance G, Cone-Wesson B, Wunderlich J, Dowell R. Speech Perception And Cortical Event Related Potentials in Children with Auditory Neuropathy. Ear Hear 2002; 23: 239-53.

7. Liberman M Ch, Gao J, He DZ, Wu X, Jia S, Zuo J. Prestin is required for electromotility of the outer hair cell and for the cochlear amplifier. Nature 2002; 419: 300-4.

8. Claros P, turcanu D, Caballero M, Costa C, Claveria MA. Claveria Jr, et cols. Hipoacusia Neurosensioral por Hiperbilirrubinema Neonatal. Acta Otorrinolaringol Esp 2003; 54: 393-8.

9. Núñez-Batalla F, Carro-Fernández P, Antuña-León ME, González-Trelles T. Incidence of hypoacusia secondary to hyperbilirubinaemia in a universal neonatal auditory screening programme based 
on otoacoustic emissions and evoked auditory potentials. Acta Otorrinolaringol Esp 2008; 59: 108-1.

10. HALL JW. ABR. Pediatric Clinical Applications and Populations. En: New Handbook of Auditory Evoked Responses. Allyn and Bacon 2007; 313-341.

11. ShapiRo SM, NakamuRa H. Bilirubin and the auditory system. J Perinatol 2001; 21 Suppl 1: S52-5.

12. Vlastarakos PV, Nikolopoulos TP, Tavoulari E, Papacharalambous $G$, Korres S. Auditory neuropathy: Endocochlear lesion or temporal processing impairment? Implications for diagnosis and management. Int $J$ Pediatr Otorhinolaryngol 2008; 72: 8: 1135-50.

13. Gallo-Terán J, Morales-Angulo C, Sánchez N, Manrique M, Rodríguez-Ballesteros M, MorenoPelayo MA Y cols. Auditory neuropathy due to the Q829X mutation in the gene encoding otoferlin (OTOF) in an infant screened for newborn hearing impairment. Acta Otorrinolaringol Esp 2006; 57 (7): 333-5.

14. Varga R, Kelley PM, Keats BJ, Starr A, Leal SM, Cohn E, KimberLing WJ. Non-syndromic recessive auditory neuropathy is the result of mutations in the otoferlin (OTOF) gene. J Med Genet 2003; 40 (1): $45-50$.

15. Starr A, Sininger V, Winter M, Derebery MU, Ota S, Michalewski HU. Transient deafness due to temperature-sensitive auditory neuropathy. Ear Hear 1998; 19: 169-79.

16. Varga R, Avenarius MR, Kelley PM, Keats BJ, BERLIN Cl, HOOD LJ Y COLS. OTOF mutations revealed by genetic analysis of hearing loss families including a potential temperature sensitive auditory neuropathy allele. J Med Genet 2006; 43 (7): 576-81.

17. Sininger Y. Starr A. Auditory Neuropathy. Singular Thompson Learing. $1^{a}$ edición. 2001.

18. McMahon CM, Patuzzi RB, Gibson WP, Sanli H. Frequency-specific eletrocochleography indicates that presynaptic and postsynaptic mechanisms of auditory neuropathy exist. Ear Hear 2008; 29(3): $314-25$
19. Berlin Ch, Morlet T, Hood L. Auditory Neuropathy / Dyssynchrony. Its diagnosis and management. Pediatr Clin N Am 2003; 50: 331-40.

20. TLumak A. Electrophysiological Responses in individuals with Auditory Neuropathy. Semin Hear 2002; 183-191.

21. Berlin CH, Hood L, Morlet T, Wilensky D, St John P, Montgomery E et al. Absent o elevated muscle reflex in the presence of normal Otoacoustic Emissions: A universal Finding in 136 cases of auditory neuropathy/Dyssynchrony. J Am Acad Audiol 2005; 16(8): 546-53.

22. Berlin Ch, Bordelon J, St John P et al. Reversing click polarity may uncover auditory neuropathy in infants. Ear Hear 1998, 19: 37-47.

23. Zeng F, Oba S, Garde S, Sininger I, Starr A. Temporal and Speech processing deficits in auditory neuropathy. Neuroreport 1999; 8; 10(16): 3429-35.

24. Rance G, McKay C, Grayden D. Perceptual Characterization of children with Auditory Neuropathy. Ear Hear 2004; 25(1): 34-46.

25. Kumar A, Jayaram M. Auditory processing in individuals with auditory neuropathy. Behavioral and Brain Functions 2005; 1: 21.

26. Narne VK, Vanaja C. Speech identification and cortical potentials in individuals with auditory neuropathy. Behav Brain Funct 2008; 31: 4: 15.

27. Rance G, Bee DE, Cone-Wesson B, et al. Clinical findings for a group of infants and young children with auditory neuropathy. Ear Hear 1999; 20: 238-52.

28. GIBSON WP, GRAham JM. Editorial: 'auditory neuropathy' and cochlear implantation - myths and facts. Cochlear Implants Int 2008; 9 (1): 1-7.

29. Rance G, Barker EJ. Speech perception in children with auditory neuropathy/dyssynchrony managed with either hearing aids or cochlear implants. Otol Neurotol 2008; 29 (2): 5-6.

30. WolfE J \& Kasulis H. Contemporary audiologic assessment for auditory dys-synchrony. The ASHA Leader 2008; 13(2): 5-6. 